\title{
Squamous cell carcinoma of the pleura following bronchopleural fistula
}

\author{
G PRABHAKAR, I M MITCHELL, T GUHA, R NORTON \\ From the Department of Cardiothoracic Surgery, Walsgrave Hospital, Coventry
}

ABSTRACT Squamous metaplasia of the pleura with malignant transformation was observed five months after the development of an empyema and bronchopleural fistula in a 58 year old woman with lingular bronchiectasis.

Squamous metaplasia of serosal surfaces, and in particular of the pleura, is uncommon. 'Metaplasia progressing to carcinoma is even less common-in fact only one report (of six cases) has been published in recent years.

\section{Case report}

A 58 year old woman was admitted in January 1986 with a tender subcutaneous swelling $7 \mathrm{~cm}$ in diameter overlying the fifth and sixth ribs in the left anterior axillary line. Some 35 years previously a bronchiectatic left lower lobe had been resected, and since then there had been occasional episodes of cough with white or green sputum. These episodes had become increasingly frequent in the previous four years, but had responded well to antibiotics. Haemoptysis had occurred twice in the six months before admission. She had had a vagotomy and pyloroplasty for duodenal ulcer in 1977, and a hysterectomy and salpingo-oophorectomy for uterine and ovarian fibroids in 1983. Laboratory investigations showed no abnormality apart from a raised erythrocyte sedimentation rate ( $78 \mathrm{~mm}$ in one hour).

At operation the mass proved to be an abscess, which was treated by drainage and resection of the underlying rib. Proteus and Streptococcus species were cultured, and a postoperative sinogram and bronchogram showed a bronchopleural fistula communicating with the abscess site, plus bronchiectasis in the lingula. There was no improvement with antibiotics and physiotherapy, and after five months an empyema was decorticated and the lingula resected. Two weeks later the patient died of secondary haemorrhage from the pleural surface. Permission for a necropsy was refused.

The excised lingula was bronchiectatic on gross examination, with pleural adhesions and obvious inflammation of the parenchyma. Microscopically there was severe bronchiectasis and chronic pneumonitis, with irregular fibrosis of the lung parenchyma. The pleura was thickened and densely fibrotic with foci of chronic inflammation; it was lined by moderately hyperplastic and keratotic squamous epithelium showing a prominent granular cell layer (fig 1). In large areas

Address for reprint requests: $\mathrm{Mr}$ I M Mitchell, Department of Cardiac Surgery, Royal Hospital for Sick Children, Glasgow G3 8SJ.

Accepted 13 July 1989 the squamous epithelium had undergone malignant transformation to in situ and invasive, well differentiated squamous carcinoma (fig 2).

\section{Discussion}

Squamous metaplasia of the pleura was described by Yoshida (cited by Crome ${ }^{2}$ ) in a 25 year old man who had undergone repeated thoracotomies for bronchiectasis. The first clear report of squamous carcinoma arising from metaplastic mesothelial cells was published in 1976.' Six patients had developed pleural tumours after extrapleural pneumothorax for tuberculosis, and histologically there was pleural fibrosis, squamous metaplasia, and squamous carci-

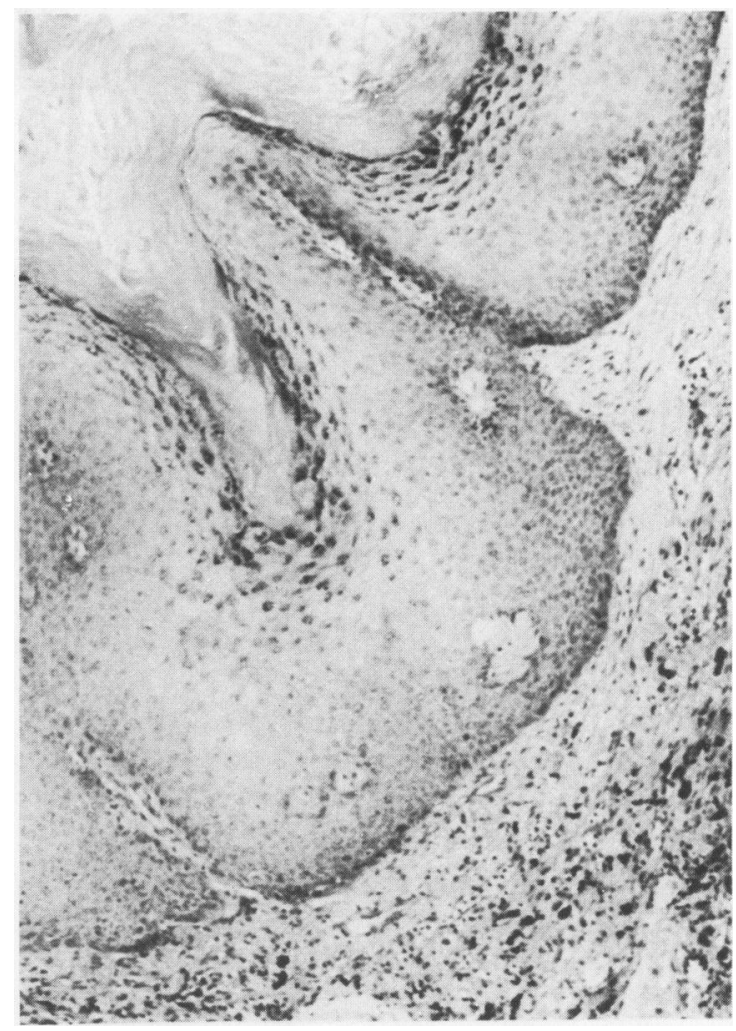

Fig 1 Hyperplastic and hyperkeratotic squamous epithelium lining the pleura and showing a prominent granular layer. 


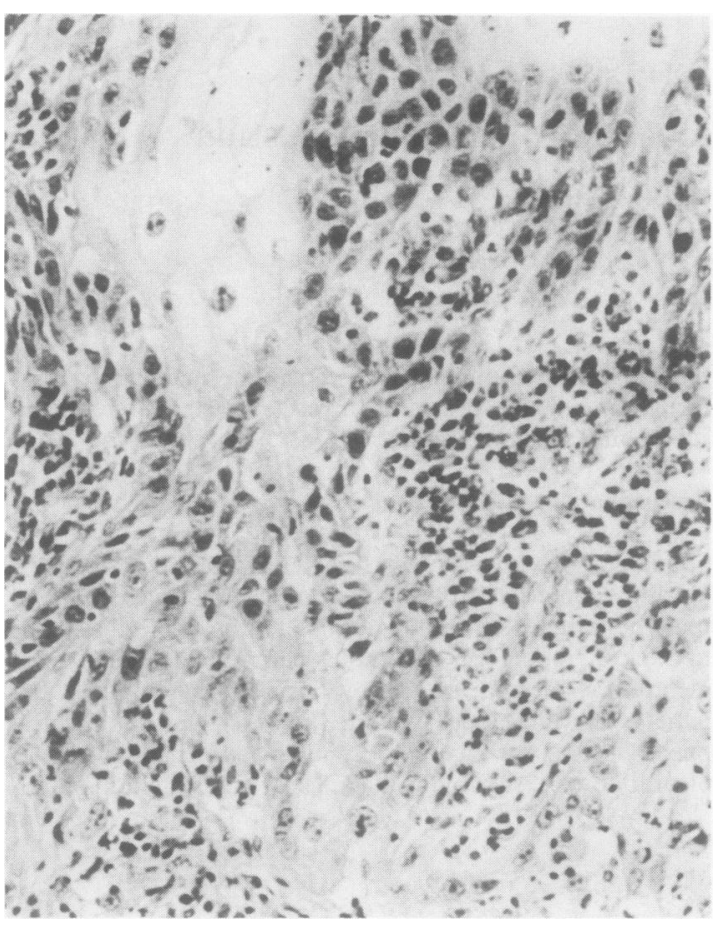

Fig 2 Squamous cell carcinoma infiltrating the pleura. noma. The interval from treatment to development of the malignancy varied from eight months to 16 years. There is $\overrightarrow{\overline{\vec{F}}}$ one reported case of squamous carcinoma of the lung complicating chronic empyema where the interval betweenc

drainage and carcinoma was 57 years. $^{3}$
In our patient the interval appears to have been as short as $\frac{\overline{5}}{\frac{5}{5}}$ five months, and the reason for the malignant transformation $\stackrel{\unrhd}{\unrhd}$ is not obvious. The change was more than mere metaplasia, since the squamous epithelium showed all four epidermal layers, with hyperkeratosis and a prominent granular layer. There appears to have been a spread of squamous epithelium $\vec{A}$ from the skin down a sinus tract, and epidermidisation would $\omega_{\sigma}$ therefore seem an appropriate term for this process. The lung could not be said to have had a cutaneous covering as no epidermal accessory structures were present.

We thank Professor B Corrin of the Brompton Hospital, $\vec{N}$ London, for reviewing the histological material and the $\vec{\circ}$ department of medical photography, Walsgrave Hospital, Coventry, for preparing the photomicrographs.

\section{References}

1 Willen R, Bruce T, Dahlström G, Dubiel WT. Squamous epithelial $\mathbb{D}$ cancer in metaplastic pleura following extrapleural pneumothorax for pulmonary tuberculosis. Virchows Arch A Pathol Anat and Histol 1976;370:225-31.

2 Crome L. Squamous metaplasia of the peritoneum. $J$ Pathol Bacteriol 1950;62:61-8.

3 Tsuji Y, Tsuhota N, Maeda K, et al. A case of thoracic empyema with bronchopleural fistula existing for fifty-seven yea 8 Histological findings of squamous cell carcinoma in resecter lung. Kyohu Gek 1984;37:768-72. 